\title{
Multienzymnetzwerke für die Feinchemie
}

\section{Journal Article}

\section{Author(s):}

Bujara, Matthias; Billerbeck, Sonja; Panke, Sven

Publication date:

2011

Permanent link:

https://doi.org/10.3929/ethz-b-000041489

Rights / license:

In Copyright - Non-Commercial Use Permitted

Originally published in:

Biospektrum 17(4), https://doi.org/10.1007/s12268-011-0061-z 


\section{Komplexe in vitro-Systeme}

\section{Multienzymnetzwerke für die Feinchemie}

MATTHIAS BUJARA, SONJA BILLERBECK, SVEN PANKE

BIOPROZESSLABOR, DEPARTMENT FÜR BIOSYSTEME, ETH ZÜRICH, BASEL, SCHWEIZ

\section{Die Produktion von Feinchemikalien beruht auf Prozessen mit mehreren \\ Reaktionsschritten. Wir arbeiten an Konzepten für die Rekrutierung, Isolierung und Optimierung von in vitro-Enzymkaskaden aus Zellextrakten für die Synthese von komplexen Zuckern.}

The production of fine chemicals relies on complex multi-step synthesis. We work on the scientific and technical challenges to recruit, insulate and optimize multi-enzyme in vitro networks from cell free extracts for the synthesis of complex sugars.

In der Weißen Biotechnologie, der Produktion von Grund- und Feinchemikalien mithilfe von Biokatalysatoren, haben sich die Produktion durch Kultivierung lebender Zellen und die Biokatalyse in zellfreien Systemen oder durch einzelne gereinigte Enzyme etabliert. Während in zellulären Systemen in der Regel komplexe metabolische Reaktionsnetzwerke nutzbar gemacht werden können, beruht die Produktion in zellfreien Systemen typischerweise nur auf wenigen Reaktionen, zumeist gar nur auf einer. Aus unserer Sicht wird die Ausdehnung der Biokatalyse auf komplexe in vitro-Enzymnetzwerke durch zwei Dinge limitiert: Das Zusammenstellen der Enzymnetzwerke ist gegenwärtig aufwendig, und die vielfältigen Interaktionen zwischen den Enzymen, den chemischen Zwischenprodukten und Elementen des zellfreien Extrakts verhindern einen effizienten Betrieb des Netzwerks. Wir arbeiten an Methoden, ein isoliertes und dynamisch optimiertes Enzymnetzwerk aus dem zellfreien Extrakt einer einzigen Kultivierung zu rekrutieren.

Komplexe, zellfreie Systeme finden unter anderem Anwendung in der zellfreien Proteinsynthese [1], der Produktion von Biowasserstoff aus Zuckern [2] und der Produktion von Feinchemikalien [3, 4]. Biokatalytische Multienzymsynthesen haben traditionell in der organischen Chemie, insbesondere im Bereich der Zuckersynthese, Eingang gefunden. Zucker gehören zu den komplexesten Biomolekülen und sind aufgrund dessen nur sehr aufwendig auf chemischem Weg zu synthetisieren. Enzyme sind typischerweise spezifisch für eine Stereokonfiguration, und unterschiedliche Stereokonfigurationen können durch den Einsatz unterschiedlicher Enzyme realisiert werden. Ein viel zitiertes

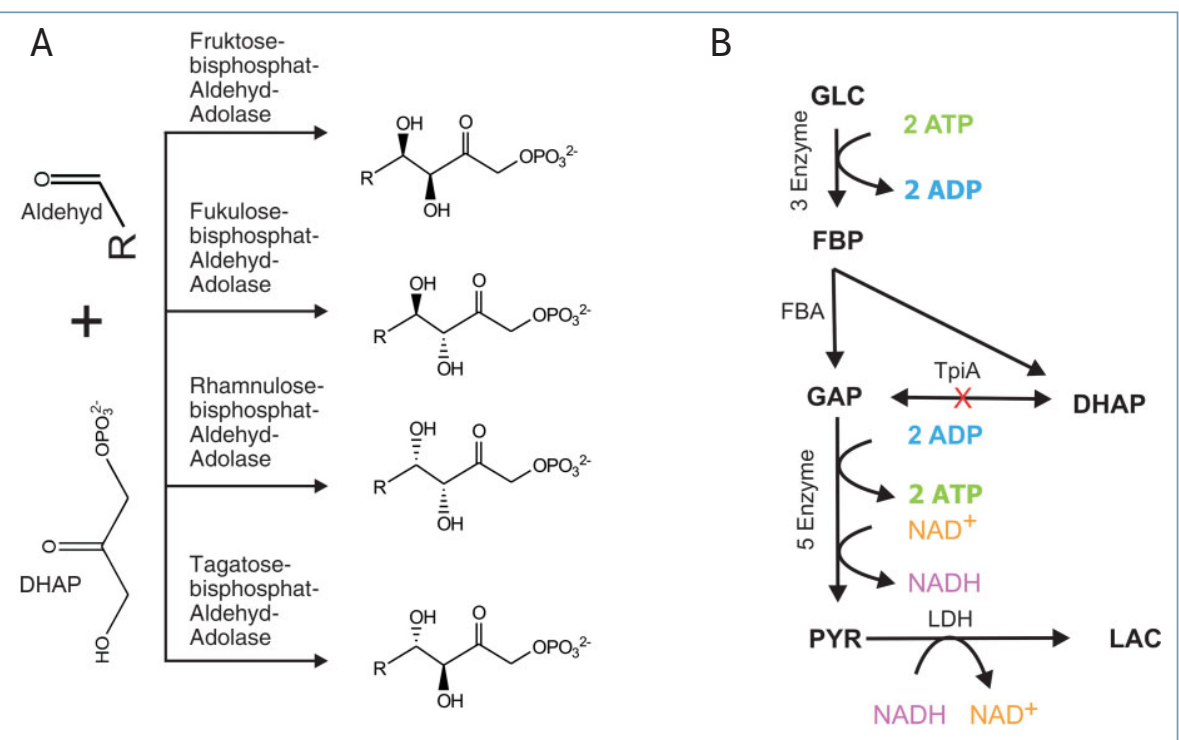

$\Delta$ Abb. 1: Herstellung von DHAP für die Zuckerproduktion. A, Kombinatorische Synthese vicinaler Diole ausgehend von DHAP mit einem stereochemisch vollständigen Satz an DHAP-abhängigen Aldolasen. B, Glykolyse-basiertes DHAP-Produktionsnetzwerk mit integrierter Kofaktorregeneration. FBA, Fruktosebisphosphat-Aldolase; TpiA, Triosephosphatisomerase; LDH, Laktatdehydrogenase; GLC, Glukose; FBP, Fruktosebisphosphat; GAP, Glycerinaldehyd-3-phosphat; PYR, Pyruvat; LAC, Laktat (weitere Erklärungen im Text). 
A

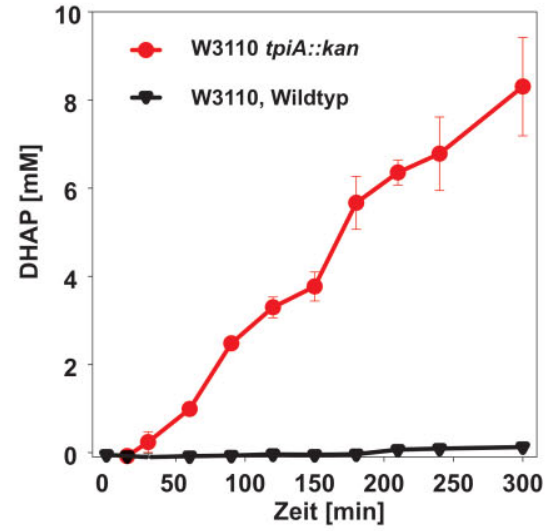

B

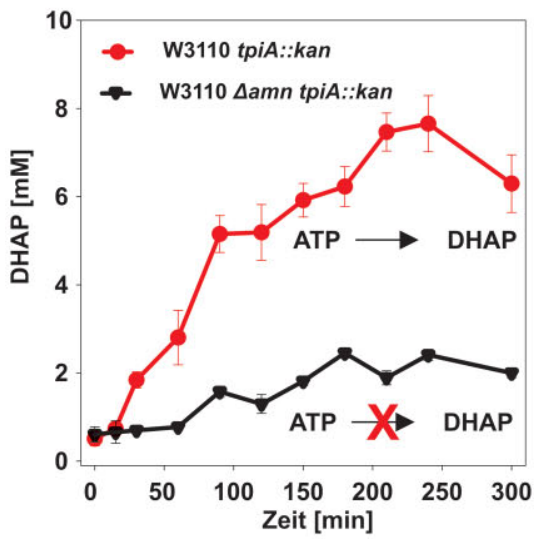

C

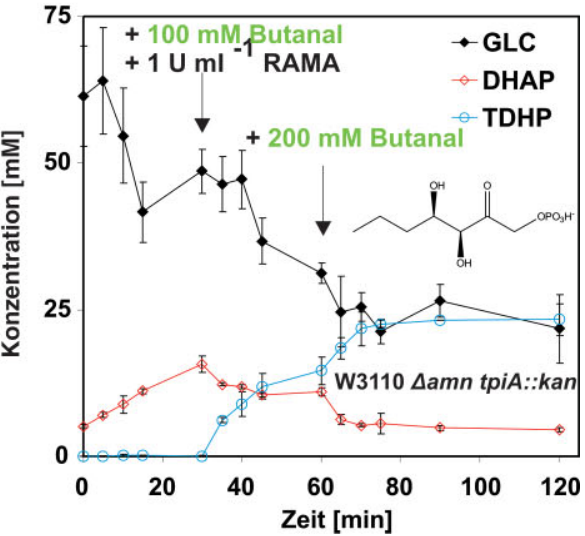

$\Delta$ Abb. 2: Produktion von DHAP in einem zellfreien Extrakt von Escherichia coli. A, Die Deletion des tpiA-Gens erlaubt die Akkumulation von DHAP aus Glukose. B, Stabilisierung des Kofaktors ATP durch Deletion des Gens für die AMP-Nukleosidase (amn). Das Experiment wurde ohne Zugabe von Glukose durchgeführt, aber mit einer hohen ATP-Konzentration (23 mM). C, Produktion von 5,6,7-Trideoxy-D-threoheptulose-1-phosphat (TDHP) aus Glukose (GLC) mittels glykolytischer DHAP-Produktion und Kaninchenmuskel-Aldolase (RAMA).

A

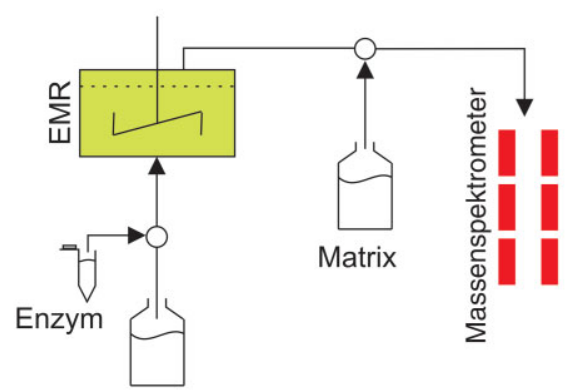

Substrateinspeisung

C

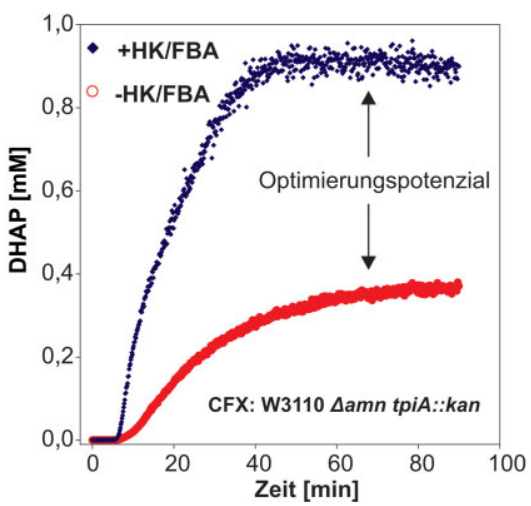

B

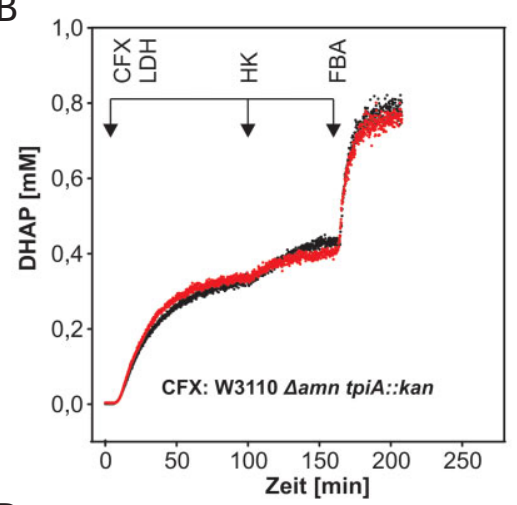

D

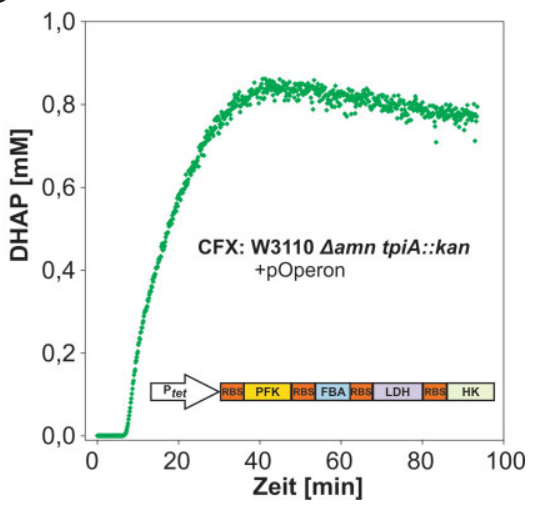

$\triangle$ Abb. 3: Optimierung der DHAP-Produktion mithilfe eines Echtzeitanalysesystems. A, Echtzeitanalysesystem zur Analyse von metabolischen in vitro-Netzwerken. Der Ausfluss eines Enzymmembranreaktors (EMR) wird kontinuierlich in einem Massenspektrometer analysiert. B, Die Reaktionen der Hexokinase (HK) und Fruktosebisphosphat-Aldolase (FBA) limitieren die DHAPProduktion in einem kontinuierlichen System. Aufgereinigte, kommerziell erhältliche HK (aus Hefe) und FBA (aus Kaninchenmuskel) wurden an zwei verschiedenen Zeitpunkten zugegeben. C, Optimierungspotenzial für die kontinuierliche DHAP-Produktion durch Erhöhung der HK- und FBA-Aktivitäten. Rot: ohne Enzymzugabe, blau: mit Zugabe von HK und FBA. D, Implementierung eines Operons steigert die DHAP-Produktion im selben Maße wie die Zugabe von gereinigten Enzymen und macht das Produktionssystem unabhängig von der Zugabe. LDH, Laktatdehydrogenase; PFK, Phosphofruktokinase; CFX, zellfreies Extrakt.

die Spaltung von Fructose-1,6-bisphosphat (FBP) in DHAP und Glycerinaldehyd-3-phosphat (GAP) (Abb. 1B). Durch Ausschalten der Triosephosphatisomerase (TpiA) kann DHAP akkumulieren, während der ATP-Bedarf des Systems theoretisch durch kontinuierliche ATP-Regeneration im unteren Teil der Glykolyse gedeckt wird. Wenn man nun zellfreies Extrakt eines Escherichia coli-Stamms einsetzt, dem die TpiA-Aktivität entfernt wurde, so akkumuliert in Batch-Reaktionen DHAP (Abb. 2A, [4]). Dieses System erfordert allerdings hohe Konzentrationen an ATP, um Glukose vollständig zu DHAP umzusetzen. Der Grund dafür ist das Vorhandensein von Enzymen im zellfreien Extrakt, die ATP (oder auch ADP oder AMP) als Substrat verwenden, aber nicht zur Produktion von DHAP beitragen. ATP kann sogar über AMP anstelle von Glukose als Substrat für die DHAP-Produktion genutzt werden, wodurch der AXP-Spiegel (AXP: Summe aus ATP, ADP und AMP) abnimmt (Abb. 2B). Als ersten Schritt in der Optimierung des Systems wurde der AXPSpiegel stabilisiert, indem das Gen für die AMP-Nukleosidase (amn), die AMP zu Adenin und Ribose-5-phosphat hydrolysiert, entfernt wurde. Dies erlaubte außerdem eine Reduktion der ATP-Zugabe um 75 Prozent [4].

Aufgrund seiner mangelnden Stabilität ist DHAP kein geeignetes Endprodukt einer Reaktionskaskade, sondern besser ein Substrat für die kombinatorische Synthese von unnatürlichen Zuckern. Die exemplarische Synthese von 5,6,7-Trideoxy-D-threoheptulose-1-phosphat (TDHP) mit dem zellfreien Extrakt der tpiA-amn-Doppelmutante zeigt, 
dass dies prinzipiell möglich ist. Als Aldehyd wurde Butanal hinzugegeben. Da die endogene E. coli-Aldolase fast ausschließlich GAP als Aldehyd akzeptiert, wurde eine kommerziell erhältliche Aldolase aus dem Kaninchenmuskel hinzugegeben, die bekannt für ihre Akzeptanz einer breiten Palette von Aldehyden ist. Mit diesem System konnten ca. 40 Prozent der bereitgestellten Glukose zu TDHP umgesetzt werden (Abb. 2C).

\section{Optimierung durch Echtzeitanalyse}

Weiterhin sollte die Produktionsrate des Systems, also die zehn Reaktionen von Glukose zu Laktat und DHAP, optimiert werden. Um Rückschlüsse auf potenziell limitierende Reaktionen ziehen zu können, müssen möglichst alle Metaboliten gemessen werden. Die Analyse von glykolytischen Metaboliten mittels Chromatografie und Massenspektrometrie stellt allerdings nach wie vor einen erheblichen Zeitaufwand dar. Um diesen Engpass zu überwinden, haben wir ein Messsystem entwickelt, das eine Echtzeitaufnahme von 15 Metabolitkonzentrationen mit einer Zeitauflösung von einer vollständigen Messung alle acht Sekunden erlaubt [8]. Das System basiert auf einem als Chemostat betriebenen Enzymmembranreaktor (EMR), bei dem der Ausfluss nach nur einem Verdünnungsschritt kontinuierlich in einem Massenspektrometer analysiert wird (Abb. 3A). Durch die Membran werden die Enzyme im Reaktorinneren zurückgehalten, während die Metaboliten passieren können. Durch den Echtzeitcharakter der Methode lässt sich das Verhalten von Metaboliten nach einer Störung, wie beispielsweise der Zugabe von einem Enzym oder einem Metabolit, online verfolgen und so quasi Online-Optimierung von Stoffwechselwegen betreiben.

Diese Analysemethode erlaubte es uns, limitierende Reaktionen in unserem Produktionsnetzwerk zu finden, indem kommerziell erhältliche, gereinigte Enzyme in den Reaktor injiziert wurden, nachdem die DHAP-Konzentration einen stationären Zustand erreicht hatte. So wurde eine kooperative Limitation in den Reaktionen der Hexokinase (HK) und der Fruktosebisphosphat-Aldolase (FBA) gefunden (Abb. 3B, C). Zur Kompensation dieses Flaschenhalses wurden die korrespondierenden endogenen E. coli-Gene in ein synthetisches Operon kloniert. Da eine feine Abstimmung der Enzymaktivitäten - insbesondere der Hexokinase - notwendig ist und eine Vorhersage von intrazellulären Enzymaktivitäten auf Basis von DNA-Sequenzen noch nicht möglich ist, mussten drei verschiedene Varianten des Operons mit verschiedener Reihenfolge der Gene und unterschiedlicher Gendosierung getestet werden. Dieser Prozess der Optimierung wurde durch die Online-Analyse erheblich vereinfacht, da in einem einzigen Experiment noch bestehende oder neu entstandene Limitationen identifiziert werden konnten [8]. Das endgültige Operon bestand aus vier Genen und erlaubte die verbesserte DHAP-Produktion ohne Zusatz von aufgereinigten Enzymen (Abb. 3D).

Die Entwicklung von neuen Methoden für die Isolierung und Optimierung von zellfreien Systemen sollten zukünftig die zuverlässige Rekrutierung von komplexeren Netzwerkstrukturen für die Biokatalyse ermöglichen, ohne auf teure und zeitintensive Enzymreinigung angewiesen zu sein. So sollten neue, ökonomische Multienzymsynthesen schnell Einzug in die feinchemische Synthese finden. Im Zuge dessen wird der "Systemcharakter“ in der Biokatalyse an Bedeutung gewinnen und letztlich stark auf die Methoden des Metabolic Engineering für lebende Systeme zurückgreifen.

\section{Danksagung}

Wir sind insbesondere Michael Schümperli, Rene Pellaux und Matthias Heinemann für ihre Hilfe bei der Implementierung der Multienzymsysteme und der Echtzeitanalysen zu Dank verpflichtet, außerdem Uwe Sauer und Nicola Zamboni von der ETH Zürich und Christian Wandrey und Marco Oldiges vom Forschungszentrum Jülich für ihre Hilfe bei der Etablierung der MS-Analyse.

\section{Literatur}

[1] Stapleton JA, Swartz JR (2010) A cell-free microtiter plate screen for improved [FeFe] hydrogenases. PLoS ONE 5:e1055 [2] Zhang YHP, Evans BR, Mielenz JR et al. (2007) High-yield hydrogen production from starch and water by a synthetic enzymatic pathway. PLoS ONE 2:e456

[3] Zhang YHP (2010) Production of biocommodities and bioelectricity by cell-free synthetic enzymatic pathway biotransformations: challenges and opportunities. Biotechnol Bioeng 105:663-677

[4] Bujara M, Schümperli M, Billerbeck S et al. (2010) Exploiting cell-free systems: implementation and debugging of a system of biotransformations. Biotechnol Bioeng 106:376389

[5] Dean SM, Greenberg WA, Wong C-H (2007) Recent advances in aldolase-catalyzed asymmetric synthesis. Adv Synth Catal 349:1308-1320

[6] Schümperli M, Pellaux R, Panke S (2007) Chemical and enzymatic routes to dihydroxyacetone phosphate. Appl Microbiol Biotechnol 75:33-45

[7] Herk T v, Hartog AF, Babich L et al. (2009) Improvement of an acid phosphatase/DHAP-dependent aldolase cascade reaction by using directed evolution. ChemBioChem 10:22302235

[8] Bujara M, Schümperli M, Pellaux R et al. (2011) Optimization of a blueprint for in vitro glycolysis by metabolic real-time analysis. Nat Chem Biol 7:271-277

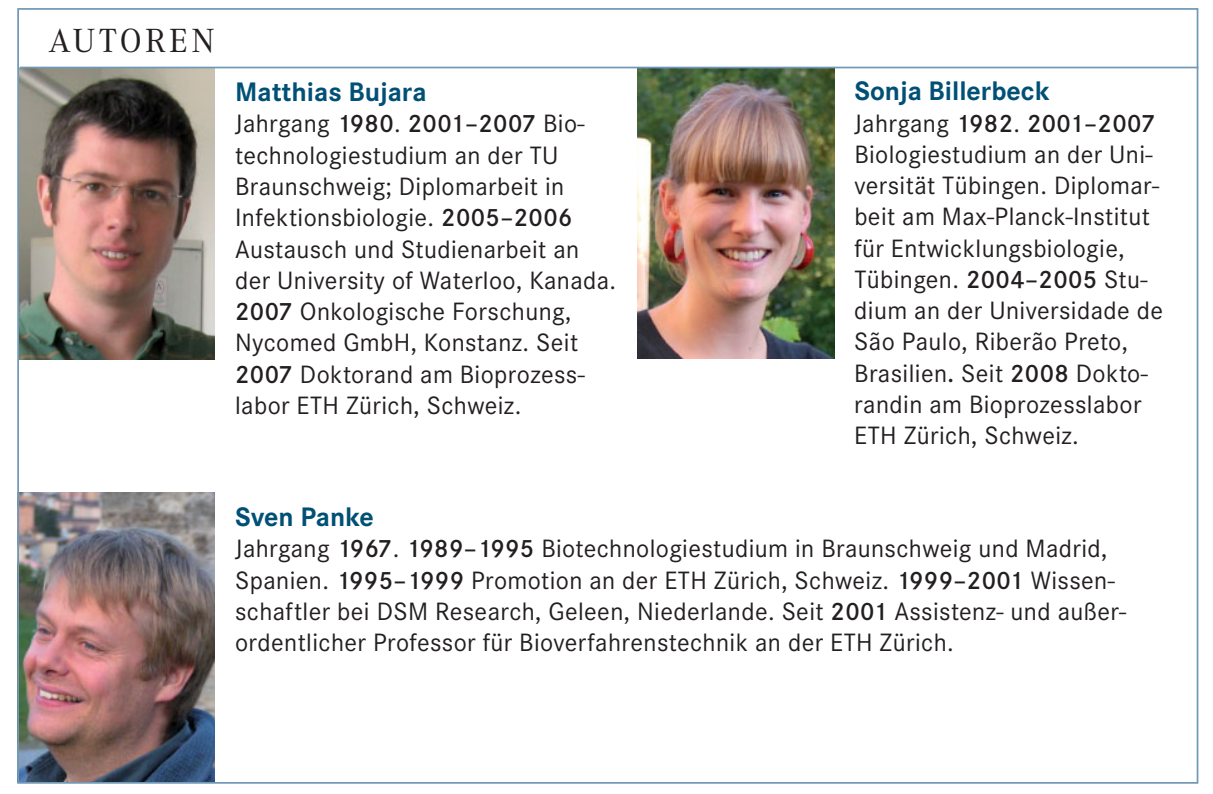

Korrespondenzadresse:

Prof. Dr. Sven Panke

Department of Biosystems Science and Engineering

ETH Zürich

Mattenstraße 26

CH-4058 Basel

Tel.: +41-(0)61-387-3209

Fax: +41-(0)61-387-3994

sven.panke@bsse.ethz.ch 